TECHNICAL NOTE

\author{
A. Bertrand \\ C. Oppenheim \\ C. Lamy \\ S. Rodrigo \\ O. Naggara \\ J.L. Mas \\ J.F. Meder
}

\title{
Comparison of Optimized and Standard Diffusion- Weighted Imaging at 1.5T for the Detection of Acute Lesions in Patients with Transient Ischemic Attack
}

SUMMARY: The high rate of normal diffusion-weighted imaging (DWI) in patients with transient ischemic attack (TIA) raises the question as to its sensitivity for detecting small ischemic lesions. We compared standard and optimized DWI in 36 consecutive patients with TIA. Optimized DWI was positive in more patients than standard DWI (19 versus 16; $P<.001$ ) and showed more lesions (56 versus $42 ; P=.002$ ). At $1.5 \mathrm{~T}$, optimizing $\mathrm{DWI}$ decreases the rate of false-negative DWI in patients with TIA.

A lthough diffusion-weighted imaging (DWI) has improved the detection of acute ischemic stroke, false-negatives are not uncommon in small infarction, brain stem location, or imaging performed early after onset. ${ }^{1}$ Increasing the magnetic field strength allows the detection of additional small bright dots on DWI in stroke patients. ${ }^{2}$ In these patients, higher sensitivity of DWI for small lesions can also be obtained at $1.5 \mathrm{~T} .^{3}$ Given that most patients are still being imaged on 1.5T MR units, our aim was to determine whether these latter results could be replicated in patients with transient ischemic attack (TIA).

\section{Technique}

MR imaging studies were performed on a 1.5T MR unit (GE Healthcare, Milwaukee, Wis). Standard DWI was acquired using a spin-echo single-shot echo-planar sequence with the following parameters: TR/ TE, 6400/86.3 ms; FOV, $240 \times 240 \mathrm{~mm}$; matrix, $128 \times 128 \mathrm{~ms} ; 20 \times$ $24 \times 6$-mm-thick contiguous sections; NEX $=2 ; b=0-1000 \mathrm{~mm}^{2} / \mathrm{s}$ along 3 diffusion directions; and acquisition time $=51$ seconds. Optimized DWI consisted of 40 3-mm-thick sections, acquired using similar parameters except for the following: $\mathrm{NEX}=3$; diffusion directions $=9$; gap $=0.3 \mathrm{~mm}$; and acquisition time $=6$ minutes.

This was a retrospective study of patients admitted to a stroke unit during a 4-month period. Fifty-three consecutive patients were referred for clinical suspicion of TIA after the exclusion of patients with isolated amaurosis fugax. Five patients were excluded because of MR contraindication and 4 others because the image quality of either standard or optimized DWI was not diagnostic, due to magnetic susceptibility or motion artifacts. Of the 44 remaining patients, all except 8 underwent standard and optimized DWI during the initial MR examination. The final study group consisted of 36 patients.

After correction of distortions, all of the images were randomly reviewed by 2 readers blinded to the clinical data, fluid-attenuated inversion recovery images, and apparent diffusion coefficient (ADC)

Received May 27, 2007; accepted after revision July 1

From the Departments of Neuroradiology (A.B., C.O., S.R., O.N., J.F.M.) and Neurology (C.L., J.L.M.), Université Paris Descartes, Centre Hospitalier Sainte-Anne, Paris, France.

Please address correspondence to Catherine Oppenheim, Departement of Neuroradiology, Université Paris Descartes, Centre Hospitalier Sainte-Anne, 1 rue Cabanis, 75014 Paris, France; e-mail: c.oppenheim@ch-sainte-anne.fr

DOI 10.3174/ajnr.A0802 maps. DWI lesions were defined as hyperintense regions after exclusion of T2 shinethrough effects due to hyperintensities on T2weighted echo-planar images $\left(b=0 \mathrm{~mm}^{2} / \mathrm{s}\right)$. Cases of discordance between readers were resolved by consensus. For each patient with lesions seen on both DWIs, a region of interest was centered in the largest area of diffusion hyperintensity (region of interest area $=28 \pm$ $18 \mathrm{~mm}^{2}$, mean $\pm \mathrm{SD}$ ) and mirrored on to the contralateral hemisphere to obtain a ratio of signal intensity (rSI) and ADC (rADC). Volume and topography of all of the additional lesions depicted on optimized DWIs were noted.

SPSS 14.0 (SPSS, Chicago, Ill) was used for statistical analysis. Agreements were assessed by calculating the $\kappa$ coefficient and its $95 \%$ confidence interval (CI) for the dichotomous data. After consensus, the number of patients with at least 1 lesion, total number of lesions, rSI, and rADC were compared between standard and optimized DWIs by using Fisher exact test for binary variables, paired Wilcoxon test for the total number of lesions, and Student $t$ test for continuous variables. A value of $P \leq .05$ was considered significant.

\section{Results}

The 36 patients consisted of 21 men and 15 women (mean age, $66 \pm 13$ years). The median duration of symptoms was 2 hours and 50 minutes (range, 1 minute to 23 hours). The median delay from onset to MR imaging was 34 hours (range, 3 hours to 8 days), with 13 patients imaged within 12 hours. TIA symptoms were as follows: aphasia $(n=8)$, motor weakness $(n=$ $18)$, isolated sensory disturbance $(n=3)$, other visual deficits $(n=3)$, brain stem symptoms $(n=3)$, and cerebellar symptoms $(n=1)$.

For the distinction between positive and negative DWIs, the 2 readers agreed in all of the cases on optimized DWI, whereas $\kappa=0.89$ (95\% CI, 0.73-1.04) for standard DWI. After consensus, a total of 56 lesions on optimized DWI and 42 lesions on standard DWI was identified, with an average of 1.6 versus 1.2 lesions per patient for optimized and standard DWI, respectively $(P=.002)$. The agreement between standard and optimized DWI was $\kappa=0.83$ (95\% CI, 0.65-1.40) for the distinction between positive and negative DWI and $\kappa=0.60$ (95\% CI, 0.41-0.91) for the number of lesions. None of the lesions seen on standard DWI were missed on optimized DWI. Of the 20 patients with normal standard DWI, 3 had lesions on 


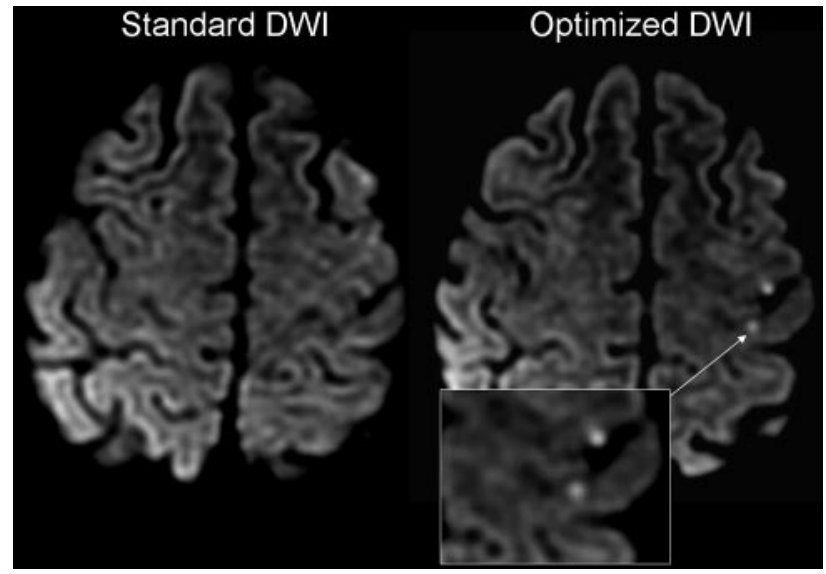

Fig 1. A 59-year-old man referred for aphasia and sensorimotor weakness of the right face and upper limb that lasted 90 minutes. Optimized DWI showed 2 punctate cortical lesions in the left middle cerebral artery territory, not visible on standard DWI. These lesions were located in the left primary sensory motor cortex, corroborating clinical symptoms.

optimized DWI (Fig 1), 2 of which were imaged within 12 hours from onset. These were frontal or temporal cortical lesions that matched clinical symptoms in 1 patient and displayed a $20 \%$ ADC decrease for the 2 others. Overall, optimized DWI improved the detection of at least 1 lesion per patient compared with standard DWI (19 versus 16; $P<.001$ ). This remained significant when the analysis was restricted to the patients imaged within 12 hours from onset $(P=.02)$. Of the 16 patients who had recent ischemia identified on standard DWI, 7 had 1-3 additional lesions on optimized DWI, located in the same arterial territory as that seen on standard DWI. Optimized DWI showed 14 additional lesions in 10 patients (Fig 2). These were small (mean $\pm \mathrm{SD}=45 \pm 41 \mathrm{~mm}^{3}$ ), preferentially located in the cortex $(n=11)$, and occasionally in brain stem $(n=2)$ or deep white matter $(n=1)$. According to the Trial of Org 10172 in Acute Stroke Treatment criteria, a vascular cause was identified in 10 patients, including 7 patients with a positive standard DWI and 1 with ischemia seen only on optimized DWI. The rSI of ischemic lesions was significantly $(P=.002)$ higher on optimized DWI $(1.77 \pm 0.60)$ than on standard DWI $(1.54 \pm 0.45)$. The rADC was similar on optimized DWI $(0.80 \pm 0.19)$ and on standard DWI $(0.82 \pm 0.15 ; P$ value not significant $)$. In patients imaged within 12 hours from onset, the rSI was still higher on optimized DWI but was no longer significantly different from rSI measured on standard DWI $(P=.08)$.

\section{Discussion}

DWI is becoming an important technique for optimum management of patients with TIA. ${ }^{4}$ The DWI sequence used in clinical practice covers the brain in less than a minute, which conforms to the time constraints of acutely ill patients with stroke. These constraints, however, are less crucial in patients with TIA, who are occasionally imaged while the deficit has already partially resolved and often after complete regression of neurologic symptoms. Increasing the acquisition time devoted to DWI to improve the detection of small ischemic lesions is, thus, conceivable. We implemented such a DWI sequence by reducing the section thickness by half to minimize the partial volume effect and increasing the number of diffusion directions and the signal

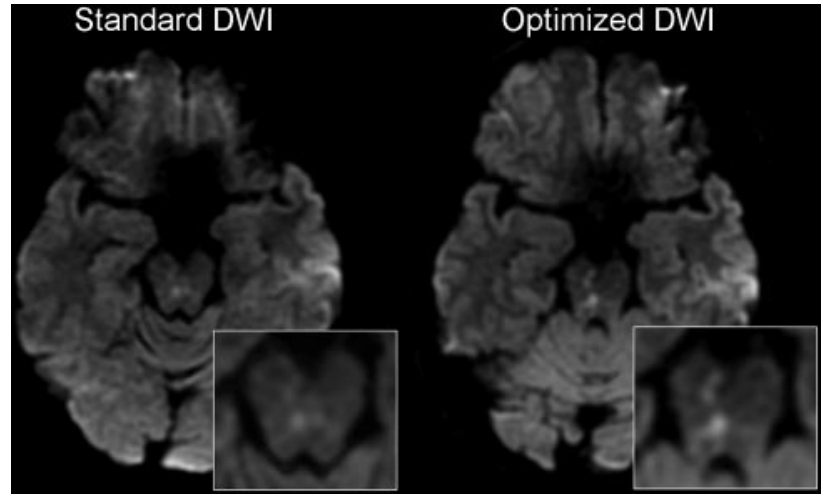

Fig 2. A 51-year-old man referred for ataxia and left facial paresthesia that lasted 20 minutes. Standard DWI demonstrated a subtle punctuate hyperintensity in the brain stem. Optimized DWI confirmed a recent ischemic brain stem lesion and revealed additional bright dots in the right cerebral peduncle, matching clinical symptoms.

intensity average to improve the angular resolution and signal intensity-to-noise ratio. These modifications improved the sensitivity for the detection of recent ischemic lesions in patients with TIA at $1.5 \mathrm{~T}$. The additional lesions identified were, without exception, small. We speculate that these lesions were not seen on the corresponding standard DWI because of the lower signal intensity and contrast-to-noise ratios, which, together with partial volume averaging of the adjacent normal tissue, precluded the diagnosis on the standard DWI. In our series of 36 patients with TIA, optimized DWI yielded approximately $8 \%$ higher sensitivity and allowed 3 patients to receive the diagnosis of acute stroke. This suggests that slight improvement of DWI parameters can result in clinically relevant findings in patients with suspected TIA. Indeed, the presence of a DWI lesion confirms the vascular origin of the deficit and is associated with a greater risk for future stroke and functional dependence. ${ }^{5}$ We extend to TIAs the results obtained by others in acute stroke patients, with a slightly different optimized DWI sequence on a $1.5 \mathrm{~T}$ unit. ${ }^{3}$ Although these authors did not report any stroke patient with bright dots exclusively visible on optimized DWI and normal standard DWI, optimized DWI showed twice as many lesions as standard DWI. In line with our findings, they reported a frequent cortical location of supplementary lesions. We speculate that the high proportion of cortical lesions that we observed may partly be due to the eddy currents distortion correction algorithm that are more accurate when at least 6 diffusion directions are acquired. The relative signal intensity ratio of lesions on optimized DWI was significantly greater than that measured on standard DWI, whereas ADC decrease $(\sim 20 \%)$ did not significantly differ on both sequences. This suggests that improving the angular resolution by using 9 instead of 3 diffusion directions does not solve the problems of ADC measurements in very small lesions.

High-field DWI MR improves the detection of small ischemic lesions in subacute stroke. However, image distortions at $3 \mathrm{~T}$ are substantial and may interfere with diagnosis. ${ }^{2}$ Until advanced acquisition strategies to reduce geometric distortion are developed and high-field MR imaging systems become widely available, a large proportion of patients with TIA will 
keep being imaged on 1.5T imaging operating systems and may, thus, benefit from optimized DWI. Optimized DWI may be a useful adjunct when facing a doubtful signal intensity change on standard DWI because of the excellent interobserver agreement that we reported. It may also be the only way to confirm a transient neurologic deficit as being ischemic when standard DWI fails to show acute lesion.

In conclusion, our study suggests that optimization of DWI acquisition parameters at $1.5 \mathrm{~T}$ increases the sensitivity of DWI for the detection of small ischemic lesions, which are the hallmark of TIA-related lesions.

\section{References}

1. Oppenheim C, Stanescu R, Dormont D, et al. False-negative diffusionweighted MR findings in acute ischemic stroke. AJNR Am J Neuroradiol 2000;21:1434-40

2. Kuhl CK, Textor J, Gieseke J, et al. Acute and subacute ischemic stroke at high-field-strength (3.0-T) diffusion-weighted MR imaging: intraindividual comparative study. Radiology 2005;234:509-16

3. Benameur K, Bykowski JL, Luby M, et al. Higher prevalence of cortical lesions observed in patients with acute stroke using high-resolution diffusionweighted imaging. AJNR Am J Neuroradiol 2006;27:1987-89

4. Kidwell CS, Alger JR, Di Salle F, et al. Diffusion MRI in patients with transient ischemic attacks. Stroke 1999;30:1174-80

5. Coutts SB, Simon JE, Eliasziw M, et al. Triaging transient ischemic attack and minor stroke patients using acute magnetic resonance imaging. Ann Neurol 2005; $57: 848-54$ 